\title{
A Dynamic Finite Element Analysis of Human Foot Complex in the Sagittal Plane during Level Walking
}

\author{
Zhihui Qian ${ }^{1}$, Lei Ren ${ }^{1,2}$, Yun Ding ${ }^{3}$, John R. Hutchinson ${ }^{4}$, Luquan Ren ${ }^{1 *}$ \\ 1 Key Laboratory of Bionic Engineering, Jilin University, Changchun, P.R. China, $\mathbf{2}$ School of Mechanical, Aerospace and Civil Engineering, University of Manchester, \\ Manchester, United Kingdom, 3 Editorial Department of Journal of Bionic Engineering, Jilin University, Changchun, P.R. China, 4 Structure and Motion Laboratory, The \\ Royal Veterinary College, University of London, Hatfield, United Kingdom
}

\begin{abstract}
The objective of this study is to develop a computational framework for investigating the dynamic behavior and the internal loading conditions of the human foot complex during locomotion. A subject-specific dynamic finite element model in the sagittal plane was constructed based on anatomical structures segmented from medical CT scan images. Three-dimensional gait measurements were conducted to support and validate the model. Ankle joint forces and moment derived from gait measurements were used to drive the model. Explicit finite element simulations were conducted, covering the entire stance phase from heel-strike impact to toe-off. The predicted ground reaction forces, center of pressure, foot bone motions and plantar surface pressure showed reasonably good agreement with the gait measurement data over most of the stance phase. The prediction discrepancies can be explained by the assumptions and limitations of the model. Our analysis showed that a dynamic FE simulation can improve the prediction accuracy in the peak plantar pressures at some parts of the foot complex by $10 \%-33 \%$ compared to a quasi-static FE simulation. However, to simplify the costly explicit FE simulation, the proposed model is confined only to the sagittal plane and has a simplified representation of foot structure. The dynamic finite element foot model proposed in this study would provide a useful tool for future extension to a fully muscle-driven dynamic three-dimensional model with detailed representation of all major anatomical structures, in order to investigate the structural dynamics of the human foot musculoskeletal system during normal or even pathological functioning.
\end{abstract}

Citation: Qian Z, Ren L, Ding Y, Hutchinson JR, Ren L (2013) A Dynamic Finite Element Analysis of Human Foot Complex in the Sagittal Plane during Level Walking. PLoS ONE 8(11): e79424. doi:10.1371/journal.pone.0079424

Editor: Jörg Langowski, German Cancer Research Center, Germany

Received January 23, 2013; Accepted September 23, 2013; Published November 11, 2013

Copyright: $\odot 2013$ Qian et al. This is an open-access article distributed under the terms of the Creative Commons Attribution License, which permits unrestricted use, distribution, and reproduction in any medium, provided the original author and source are credited.

Funding: This work was supported by the International Cooperation Project of National Natural Science Foundation of China (No. 50920105504), a Biotechnology and Biological Sciences Research Council Grant (No.BB/H002782/1), the Project of National Natural Science Foundation of China (No. 51105167) the Fundamental Research Founds of China (No. Z201105), the Key project of National Science Foundation of China (No, 51290290 \& 51290292)? the postdoctoral project (No, 2013M530985) and the scientific and technological development planning project of Jilin Province (No, 20130522187JH). The funders had no role in study design, data collection and analysis, decision to publish, or preparation of the manuscript.

Competing Interests: The authors have declared that no competing interests exist.

*E-mail: lei.ren@manchester.ac.uk

\section{Introduction}

The human foot is a very complex structure comprising numerous bones, muscles, ligaments, synovial joints and other tissues. As the only body component in contact with the ground, it plays multiple crucial roles in attenuating ground impacts, supporting against gravity, maintaining locomotor stability and transmitting or generating propulsive power during locomotion [1-3]. Over recent decades, a large number of experimental and computer simulation studies have investigated how the human foot complex functions in locomotion [4-9].

Of the numerous mathematical modelling approaches used for analyzing biomechanical foot functions, finite element (FE) analysis offers a powerful tool to assess the internal loading conditions of the foot musculoskeletal structure during human locomotion $[5,10]$. It can provide valuable estimates of stress and strain distributions in the foot bones and soft tissues, which are usually not measurable in vivo. Lemmon et al. [11] used a two dimensional (2D) FE foot model to study the effects of the shoe insole on therapeutic footwear biomechanics. A stress analysis study was conducted on both normal and neuropathic feet using a 2D foot FE model derived from a lateral X-ray image [12]. Jacob et al. $[13,14]$ conducted quasi-static analyses of different gait phases using a three-dimensional (3D) foot FE model to investigate tarsal bone degeneration in diabetes and Hansen's disease. A 3D FE foot model with detailed bone and soft tissue representations was constructed by Gefen et al. [5], in which quasi-static analyses were performed for six different instantaneous gait phases. A subject-specific 3D FE model including detailed foot structure geometry and non-linear material properties was also developed [15-17] with demonstrated potential for medical applications. Wu [18] built a 2D model to investigate the foot bone and muscle stresses resulting from plantar fasciotomy and plantar ligament injuries. Recently, a 2D FE foot model was integrated with a multi-body musculoskeletal model to simulate tissue mechanics and musculoskeletal movements simultaneously [19,20], in which prescribed kinematic boundary conditions were used to drive the foot model.

Due to the complexity of the human foot musculoskeletal structure, most previous FE models involved over-simplified loading and boundary conditions. Moreover, the analyses were static or quasi-static in nature. Yarnitzky et al. [21] developed a two dimensional hierarchal foot model, which coupled an analytical rigid body foot model with a local $\mathrm{FE}$ analysis, to study the internal deformations and stresses of heel and metatarsal head 
regions in real-time. In the rigid body model, simplified bone structure was used, by assuming to have a rotational axis in the center of cuneiform, to provide loading and boundary conditions for the FE analysis, which was quasi-static in nature. So far, there are very few foot modelling studies using explicit FE simulation [22-24]. One such investigation into foot locomotor biomechanics is that by Dai et al. [25], which examined the effect of sock wearing on plantar pressures, rather than foot-ground interactions. In their study, the load was assumed to be constant throughout the stance phase and kinematic constraints were used to define the foot motion. Simplified loading and boundary conditions and excessive constraints may lead to poor prediction of foot loading conditions during natural human movements. As a result, although quasistatic foot function has been reasonably represented, modelling and simulation studies to date have illuminated only limited details about the more realistic, dynamic response of the foot complex during human locomotion.

The objective of this study is to develop a subject-specific dynamic FE human foot model based on individualized medical imaging data and loading conditions derived from gait measurements on the same subject. No prescribed kinematic conditions are used to constrain the foot motions, which allows for the representation of natural foot movements during human locomotion. The model's validity was rigorously tested by comparing the predicted foot bone motions, ground reaction forces, center of pressure $(\mathrm{CoP})$ and plantar pressure distributions with the measured data.

\section{Materials and Methods}

\section{Ethics Statement}

This study was approved by the Institutional Review Board Committee of Jilin University, Changchun, China, and the subject gave his written informed consent (as outlined in PLOS consent form) to participate in the CT scanning and gait measurements.

\section{Finite Element Foot Modeling}

The foot geometric model was constructed from medical CT images (Lightspeed 16, General Electric Company, Fairfield, U.S.A.), which were obtained by scanning the right foot of a healthy male subject (age: $27 \mathrm{yrs}$, weight: $75 \mathrm{~kg}$; no history of lower limb injury or foot abnormalities) in the neutral unloaded position with a $1.5 \mathrm{~mm}$ slice interval, $220 \mathrm{~mA}$ and $120 \mathrm{kV}$. A 2D cross section in the sagittal plane along the first ray of the foot was taken to construct the geometric model (similar to [19,20]) using the SolidWorks software (Dassault Systèmes SolidWorks Corp., U.S.A.). The 2D planar model, as shown in Fig. 1, consisted of four segments. The bones other than the phalanges were considered as one segment. All the phalanges were represented as one separate part, which could move freely with respect to the metatarsal bones. The other two segments were soft tissues wrapping around the foot bones and the cartilages between the phalanges and the metatarsal bones. The geometric model was then imported into and assembled in the FE software package ABAQUS (Simulia, Providence, U.S.A.).

An analytical rigid flat plate was used to simulate the ground support. Interaction between the foot and the ground was modeled as a kinematic contact with a frictional coefficient of 0.6 [16]. The kinematic constraint method uses a predictor/corrector algorithm in each time increment to strictly impose contact constraints (no substrate penetration is allowed) and to conserve momentum [26]. The constraints are imposed on the global equations by a transformation of the nodal displacement components of the slave nodes along the contact interface. In the corrector phase, the depth of each slave node's penetration, its associated mass, and the time increment are used to calculate the resistant force required to prevent penetration. In the horizontal direction, the finite sliding method, allowing for arbitrary separation, sliding and rotation of the contact surfaces, was employed [26]. The bones and soft tissues were meshed using a total number of 4259 quadrilateral elements, which was determined through a convergence analysis by gradually increasing the mesh density until the deviations in the estimated stresses reached $<5 \%$ [5]. The soft tissue of the foot was assumed to be homogeneous, isotropic and linearly elastic [27]. A Rayleigh material damping coefficient (alpha) 6.7 was defined to represent the viscous behavior of the foot soft tissue in ABAQUS [26]. The damping coefficient was determined using a trial and error process to minimise the ground reaction force oscillations generated after the heel strike in the simulation. The metatarsalphalangeal joint in the sagittal plane was connected using hyperelastic cartilages [18]. The material properties of all structures modelled in this study are listed in Table 1, taken from the literature as cited. Plane stress section thickness was set to $60 \mathrm{~mm}$, an approximate foot width of the subject, to achieve a reasonable foot mass in the simulation. This resulted in a total foot mass of $1.025 \mathrm{~kg}$, about $1.37 \%$ of the subject's body mass, which is very close to that estimated using a cadaver-based anthropometric regression method [28].

The vertical and horizontal forces and the net muscle moment for the ankle joint over the whole stance phase were calculated using the inverse dynamics method [28] based on the measured segmental motions and the ground reactions (see Gait Measurements in the next section). The time traces of these ankle joint forces and moment were then used as inputs to the FE model as the dynamic loading conditions by applying them to the middle of the talus bone as the only driving forces and moment. The ground was fixed and the foot was allowed to move freely with respect to the ground without any prescribed kinematic constraints. The initial conditions of the system, including the horizontal and vertical ankle joint velocities $v_{x}, v_{y}$, foot angle $\theta_{z}$ and angular velocity $\omega_{z}$, were determined based on the measured motion data at heel-strike. The dynamic FE simulations were conducted using the ABAQUS/Explicit module to calculate the dynamic foot responses over the entire stance phase from heel-strike until toeoff. The simulation time period was set as identical to the gait measurement. The segmental inertial properties (mass, center of mass and moments of inertia) were automatically calculated by the ABAQUS software based on the model geometry and the material properties. The stability of the explicit dynamic simulation was verified by ensuring that the change of the total system energy of the foot complex was well within $1 \%$ after the whole simulation period.

\section{Gait Measurements}

Three-dimensional gait measurement was conducted to inform and validate the FE modelling. The same healthy male subject was used as in the modelling part of the study (see preceding section). A 12-camera infrared motion analysis system (Qualisys, Sweden) was used to capture the 3D motions of the lower limb segments and the foot segments at $150 \mathrm{~Hz}$. A six force plate array (Kistler, Switzerland) was used to record the $3 \mathrm{D}$ ground reactions at $1000 \mathrm{~Hz}$ and a 1-meter-long pressure plate (RSscan, Belgium) was used for foot pressure distribution recording at $250 \mathrm{~Hz}$. All three systems were digitally synchronized. A set of specially designed infrared reflective marker clusters was used (similar to [29]) to capture the delicate 3D multi-segment foot motions (see Fig. 2). The calibrated anatomical system technique [30] was used to determine the anatomical landmarks. The subject was instructed 

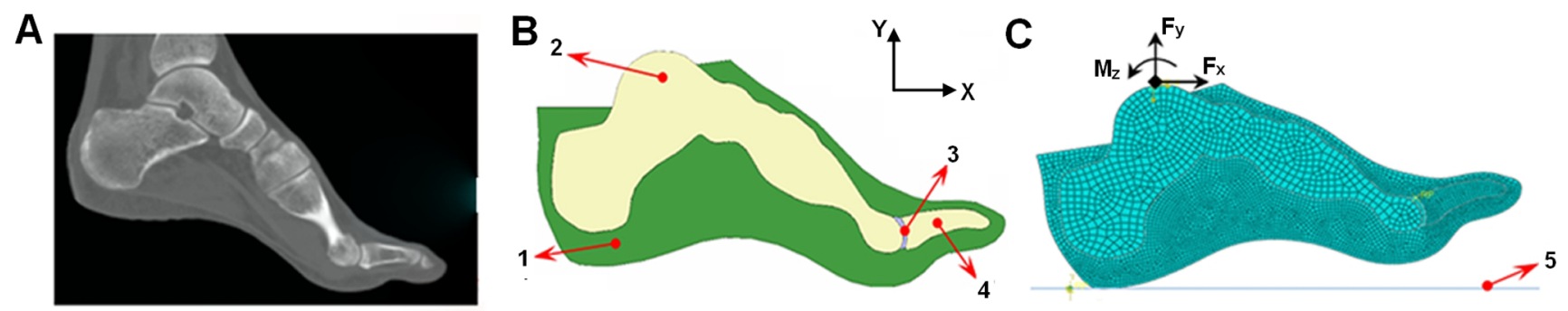

Figure 1. The 2D finite element modelling of the human foot complex. (A) The foot CT image along the first ray. (B) The 2D foot geometric model based on the CT image. (C) The 2D finite element foot model. (1. soft tissue, 2. bone structure except phalanges, 3. cartilage, 4. phalangeal bones, 5. ground surface)

doi:10.1371/journal.pone.0079424.g001

to walk barefoot at normal walking speed along a level indoor walkway. The trials were repeated ten times to ensure a representative gait pattern was obtained.

The measurement data were processed using GMAS software (the upgraded version of SMAS software), a MATLAB based software package for 3D kinematic and kinetic analysis of general biomechanical multi-body systems [28,31]. The marker data were filtered using a low pass zero lag fourth-order Butterworth digital filter with a cutoff frequency of $6.0 \mathrm{~Hz}$. The ankle joint forces and moment were calculated using inverse dynamics [28], and were used as the only muscular control variables for the foot FE model (see the FE Foot Modelling section).

\section{Error and sensitivity analysis}

The prediction accuracy of the FE foot model was quantified using the root mean square error (RMSE) and the relative root mean square error (rRMSE) ([32,33]) between the model predictions and the measurement data, which included the horizontal and vertical ground reaction forces, center of pressure, foot and phalanx rotation angles, and vertical pressures at heel and metatarsal regions over the whole stance phase. Sensitivity analysis was also conducted to investigate the effect of material properties, the frictional coefficient and the damping coefficient of the linear bulk viscosity on the model prediction results. The material properties analysis included four cases in which we changed the Young's modulus $(E)$ of the linear elastic soft tissue by $+20 \%$, $+10 \%,-10 \%$ and $-20 \%$ from the baseline value (1.15 MPa), and also two different types of non-linear material property representations (Ogden hyperelastic based on [34] and polynomial hyperelastic based on [16]). The frictional coefficient analysis included four cases in which we changed the frictional coefficient of the foot-ground contact by $+10 \%,+5 \%,-5 \%$ and $-10 \%$ from the baseline value (0.6). Whereas, the damping coefficient $b$ of the linear bulk viscosity was changed from its system default value of 0.06 to $2.0,4.0,6.0$ and 8.0 respectively in the sensitivity analysis.
Additionally, in order to compare the difference between the static FE analysis and dynamic FE analysis, quasi-static FE simulations were conducted using the same FE foot model configuration defined in FE Foot Modeling section for the dynamic FE analysis. The quasi-static FE analysis was conducted at three representative instants of time over the gait cycle, i.e. just after the heel strike (0.03 second), around the middle stance $(0.19$ second) and just before the toe off ( 0.44 second). The calculated ankle joint forces and moment at each representative instant of time were used as the loading conditions for the static simulation of each case. The calculated peak plantar pressures in the heel, metatarsal and toe regions were then compared with the peak pressure values in the three regions obtained from the dynamic FE simulation at each simulated instant of time respectively.

\section{Results}

The ankle joint forces and moment (see Fig. 3A and 3B) as well as the initial condition data from a representative gait cycle (walking speed $1.58 \mathrm{~ms}^{-1}$ ) were used as inputs to the dynamic FE simulation. The obtained simulation results are shown in Fig. 3C to $3 \mathrm{~F}$. The predicted horizontal and vertical ground reaction forces over the stance phase are compared with the force plate data in Fig. 3C. The predicted ground reaction forces showed good agreements with the measured data in terms of the general trend with rRMSEs of $13 \%$ and $10 \%$ respectively (see Table 2). However, some apparent oscillations were observed especially in horizontal components in the early stance. Fig. 3D shows the predicted CoP trajectory over the stance phase compared with the measured force plate data. Although there are some small fluctuations during early stance, the predicted CoP shows a good agreement with the measurement data throughout most of the stance phase. The major discrepancy is in the late stance phase just before toe-off, where the FE model predicts a different CoP trend with a larger displacement than the measured data. Fig. $3 \mathrm{E}$ and $3 \mathrm{~F}$ show the predicted foot rotation angle and phalanx rotation angle

Table 1. The material properties and element types used in the foot finite element model.

\begin{tabular}{|c|c|c|c|c|c|}
\hline Component & Element type & Young's modulus (MPa) & Poisson's ratio & $\begin{array}{l}\text { Mass density } \\
\left(\mathbf{k g} / \mathbf{m}^{3}\right)\end{array}$ & Reference \\
\hline Soft tissue & quadrilateral & 1.15 & 0.49 & 937.0 & {$[27]$} \\
\hline Bone structure & quadrilateral & 7300 & 0.30 & 1500 & {$[25]$} \\
\hline Phalanges & quadrilateral & 7300 & 0.30 & 1500 & {$[25]$} \\
\hline Cartilage & quadrilateral & 10, Neo-Hookean hyperelastic & $0.49, C_{10}=E /(4(1+v))$ & 2000 & {$[18]$} \\
\hline
\end{tabular}


A

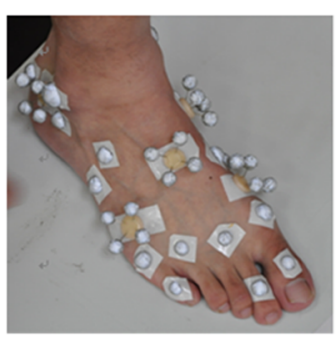

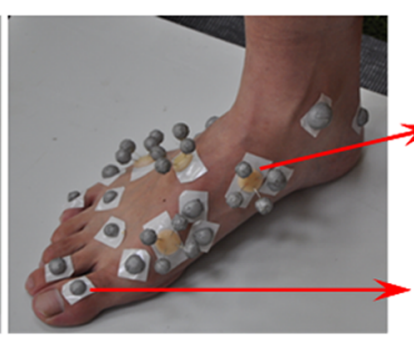

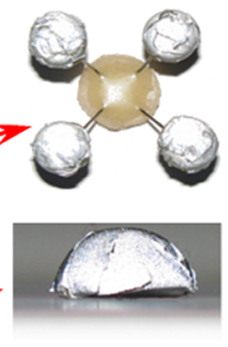

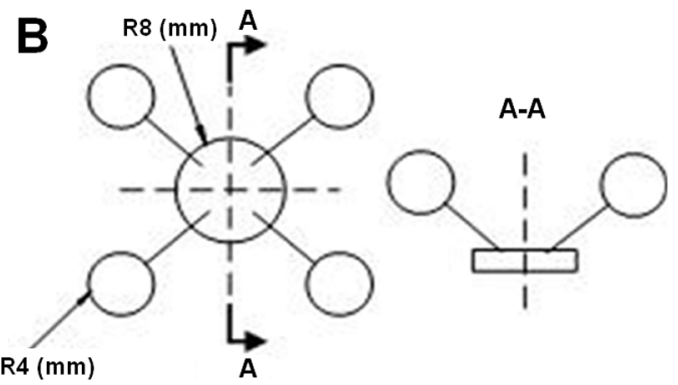

Figure 2. The infrared marker cluster system used in this study to capture 3D foot motions. (A)The foot was divided into five segments including hindfoot, midfoot, medial and lateral forefoot and toes. A set of thermal plastic plates, each carrying four infrared markers was mounted firmly on each segment to capture the segmental motions. A number of hemispherical infrared markers were also attached on the anatomical landmarks. (B) The configuration of the rigid marker cluster and the hemispherical marker. doi:10.1371/journal.pone.0079424.g002

in the sagittal plane compared with the measured data. The model predictions match the measurement data well with relative RMSEs of $9.2 \%$ and $9.3 \%$ respectively, except for some discrepancies in the early stance phase.

The predicted von Mises stress distributions on the foot at 10 typical instances of time over the stance phase are shown in Fig. 4. The location with the highest von Mises stress moved predominantly from the heel region to the toes over the stance phase. The peak plantar pressure changes at the heel medial and $1^{\text {st }}$ metatarsal regions over the whole stance phase are shown in Fig. 5. The FE simulation predicted peak plantar pressures of $0.17 \mathrm{MPa}$ and $0.19 \mathrm{MPa}$ at the heel medial and the $1^{\text {st }}$ metatarsal regions respectively, which are close to the peak pressure records (0.17 $\mathrm{MPa}$ and $0.21 \mathrm{MPa}$ ) measured by the pressure plate. However, there are some discrepancies on the timings of the peak pressures. The predicted peak plantar pressures at both the heel medial and $1^{\text {st }}$ metatarsal regions occurred about $10 \%$ of the stance phase later than in the measured data for walking.

Table 2 lists the RMSE and rRMSE results of the sensitivity analysis of foot material properties,the foot-ground frictional coefficient and the damping coefficient of the linear bulk viscosity. It can be seen that, for linearly elastic material, decreasing the Young's modulus of the foot soft tissues to $20 \%$ made no any particular improvement on the simulation results. While the prediction accuracy in horizontal ground reaction force and peak plantar pressures in the heel medial and $1^{\text {st }}$ metatarsal regions have been improved slightly by increasing the Young's modulus. Representing the material property of the soft tissue using either of Ogden or polynomial hyperelastic formulations did not lead to any improvement on simulation results compared to the linear elastic representation, except for the case of CoP for the polynomial hyperelastic representation, where the prediction error is decreased slightly compared to the baseline result using the linear elastic material property. Our sensitivity analysis of the frictional coefficient shows that the ground reaction force predictions are slightly improved by either increasing or decreasing the frictional coefficient. However, this leads to poor prediction in the foot and phalanx rotation angles, especially when decreasing the frictional coefficient. The RMSE and rRMSE results of the damping coefficient sensitivity analysis showed that the oscillation in the ground reaction forces can be significantly reduced by changing this parameter and the best prediction results are obtained when $b=6.0$. The calculated peak plantar pressures at three representative instants of time in the gait cycle by using the dynamic FE simulation were compared in Table 3 with the results obtained by using the static FE simulation. In the early stance phase (at $0.03 \mathrm{~s}$ ), the dynamic FE simulation predicted a peak plantar pressure of $0.134 \mathrm{MPa}$ at the heel medial region, which is about $10 \%$ closer to the measured $0.14 \mathrm{MPa}$ peak pressure than the static FE simulation. While in the middle stance, the quasi-static simulation provides slightly better prediction than the dynamic simulation in the heel medial region. However, in the $1^{\text {st }}$ metatarsal region, the dynamic FE simulation provided much better result than the static FE simulation in the middle stance, which is about 33\% closer to the measured peak pressure data. In the late stance, both dynamic and static simulations predicted much higher peak plantar pressures than the measurement data.

\section{Discussion}

In this study, we have developed a subject-specific 2D FE foot model that dynamically simulates the mechanics of human foot structure during normal walking. Our dynamic simulation of the entire stance phase produced visually realistic results and a compelling match with measured ground reaction forces, foot rollover angle, phalanx rotation angle, CoP displacement and plantar pressure changes in most of the stance phase, indicating that the model is reasonably valid with some exceptions noted below.

The major discrepancies between our simulation results and measurement data can be explained by the limitations and assumptions of the model. Apparent fluctuations were observed in the predicted ground reaction forces especially in the horizontal component in the early stance phase just after heel-strike. These periodic vibrations are probably due to the foot-ground impact at heel-strike, which may be more prominent at a faster walking speed, as adopted in the representative gait trial used in this study. It appears that the large kinetic energy induced by the heel-strike impact could not be effectively absorbed and attenuated immediately after heel-strike as in actual human feet [35]. The material properties and frictional coefficient sensitivity analyses suggests that this problem could not be fully remedied by changing the Young's modulus of the linear elastic material, by representing the material properties using nonlinear hyperelastic models, or by varying the foot-ground frictional coefficient (see Table 2). This may be due to the simplified foot structure in the modelling. Another possible explanation is the numerical effect. A previous study on the foot/sock-insole interaction [25] using the same explicit algorithm produced the similar oscillation in the simulated shear force time histories. Our sensitivity analysis on the linear bulk viscosity defined in the integrator of the explicit solver showed that a substantial part of the oscillation is due to the numerical effect of the dynamic integration and a proper tuning of the linear 

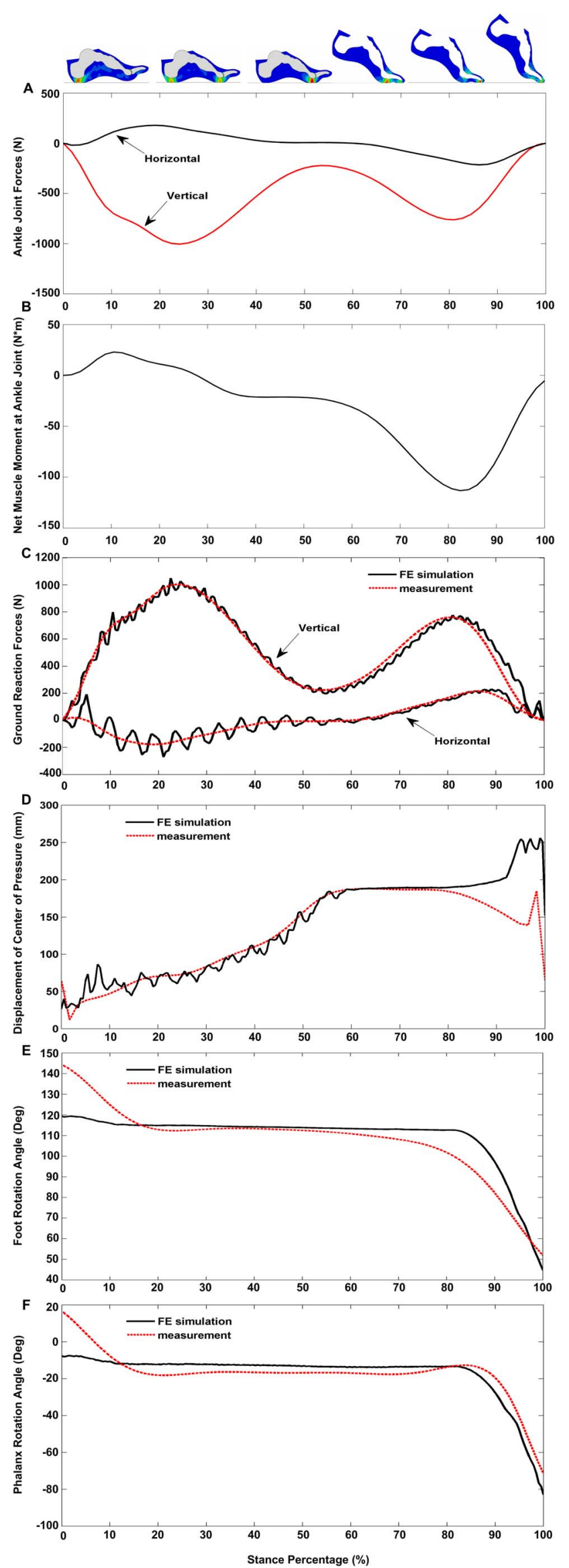

Figure 3. The FE model inputs and the FE simulation results over the whole stance phase compared with the measurement data. (A) Ankle joint force inputs. (B) Ankle moment input. (C, D, E and F) The FE simulation results compared with the measurement data. doi:10.1371/journal.pone.0079424.g003

bulk viscosity can significantly reduce the force fluctuations in both vertical and horizontal directions. It's noteworthy that there is still some small oscillations present in the horizontal force component after the linear bulk viscosity is tuned. This may be due to the contact algorithm used in the ABAQUS software to handle the horizontal contact mechanics. This strongly suggests that investigation is needed in the future to explore the effect of contact algorithms on model predictions.

Additionally, our simulations overestimated the CoP displacement in late stance (see Fig. 3D). This may be because the simulations predicted excessive toe rotations before toe-off, which made the phalangeal bones rotate around the toe tip in the late stance, thus resulting in a larger $\mathrm{CoP}$ displacement. In contrast, during normal human walking the phalangeal bones mainly stay in contact with the ground until toe-off. This discrepancy may be explained by the absence of ankle plantarflexor muscle activations in the model. The "windlass mechanism" induced by the ankle plantar-flexor contractions may help to stretch the foot plantar fascia [36] and hereby keep the toes in contact with the ground during late stance.

The FE model predicted foot rollover angles and phalanx rotation angles in the sagittal plane reasonably well, but with major discrepancies in the early stance phase (in the first $10 \%$ of the stance phase). This is probably due to the error in setting the initial angle values at heel strike. The gait measurement data were used to define the initial angles, which used the reflective markers attached on the subject's foot skin surface. In contrast, in the FE foot model, node points on the bones were used to define the rotation angles. Although our best effort was made to match the marker positions and node positions, some apparent differences still persist. This may be particularly difficult at heel strike due to the possible large skin movement artifacts induced by the footground impact. X-ray cameras or bone-mounted motion analysis markers would be needed to reduce this error.

The peak plantar pressures from the FE model predictions compared well with the pressure plate measurement data at both heel medial and $1^{\text {st }}$ metatarsal regions, but with a time delay of about $10 \%$ stance phase. This may be due to the simplifications in the model construction. In this study, the complex 3D foot structure is considerably simplified as a 2D planar model extruded with a width to match the total foot mass. This may have lead to discrepancies in dynamic structural responses, which may have resulted in a phase shift in peak pressures. In addition, the lack of consideration of sesamoid bone in the modelling may lead to underestimation of the peak plantar pressure at the $1^{\text {st }}$ metatarsal region and may also cause some delay in the plantar pressure response. Moreover, the soft tissue material properties may also play a role in determining the plantar pressure distribution. In order to reduce the complexity of the explicit FE simulation, the soft tissues were modelled as homogeneous and isotropic elastic materials in this study, was an assumption adopted by most of the previous FE foot models [12-15,18,25,27,37,38]. However, an experimental study [39] showed that the foot plantar soft tissue demonstrated strong anisotropic, time-dependent and viscoelastic material behavior. Inappropriate material property representations may lead to large errors in plantar pressure predictions, which are also suggested by the results of our sensitivity analysis of material properties. 


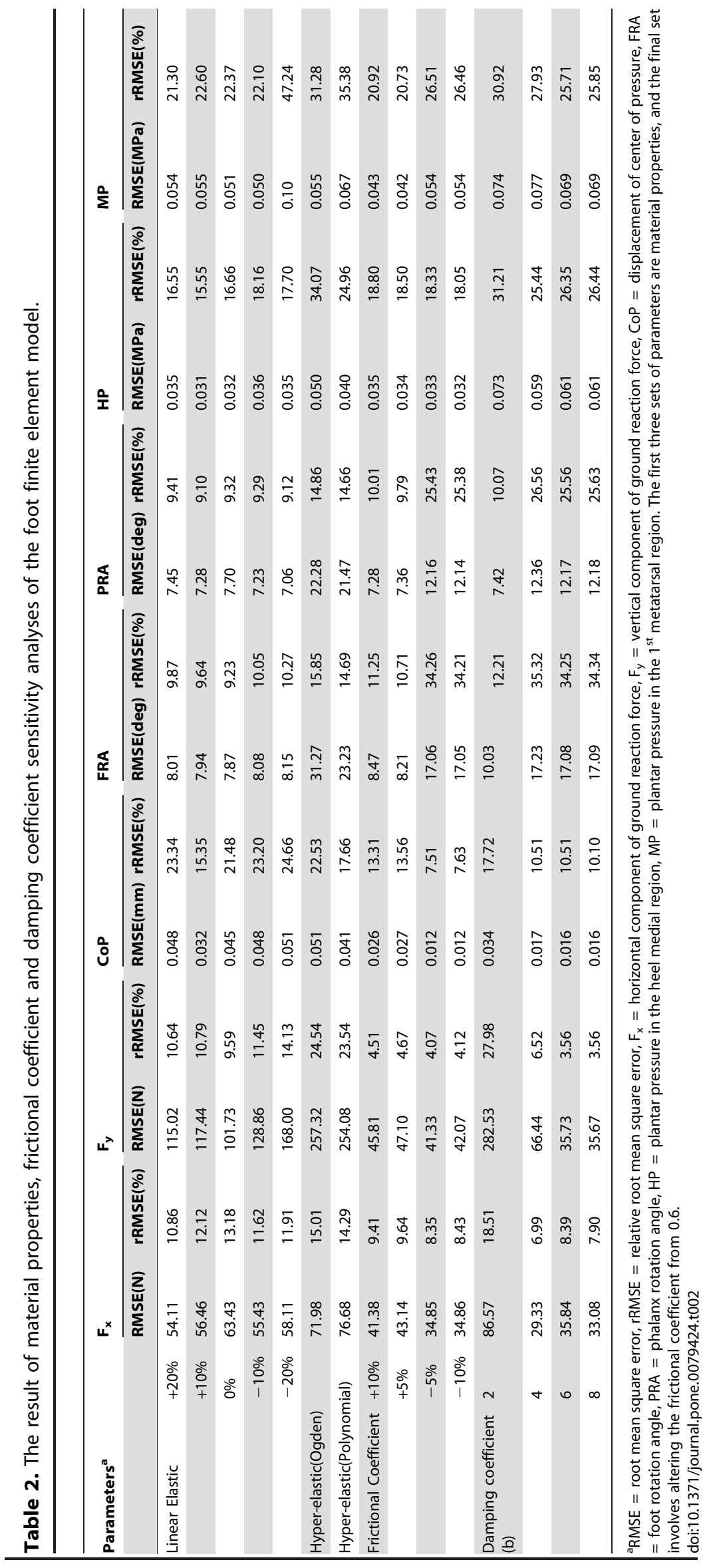




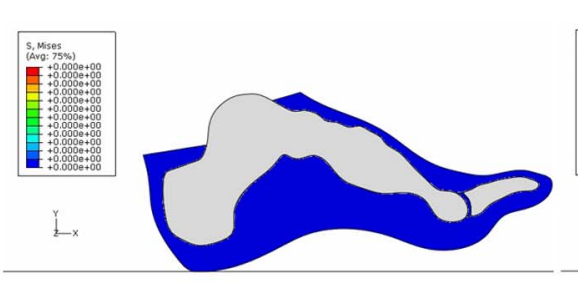
$\mathrm{t}=0$ (s)

.

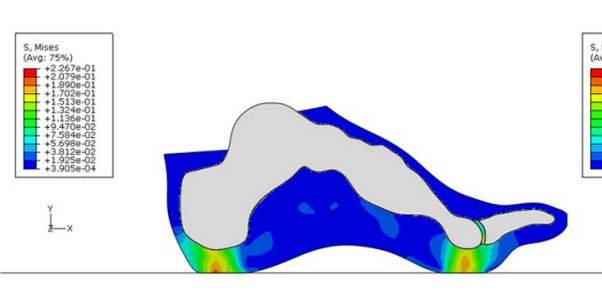

$\mathrm{t}=0.19$ (s)

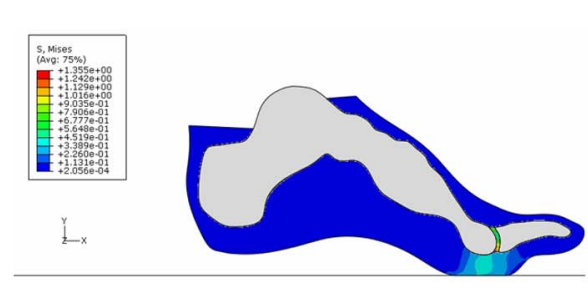

$\mathrm{t}=0.40(\mathrm{~s})$

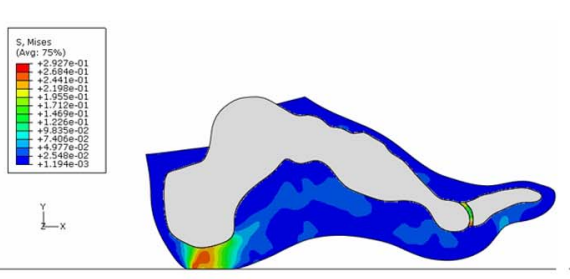

$\mathrm{t}=0.03(\mathrm{~s})$

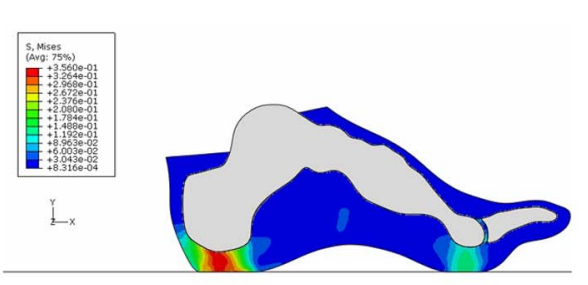

$\mathrm{t}=0.08(\mathrm{~s})$ (s)

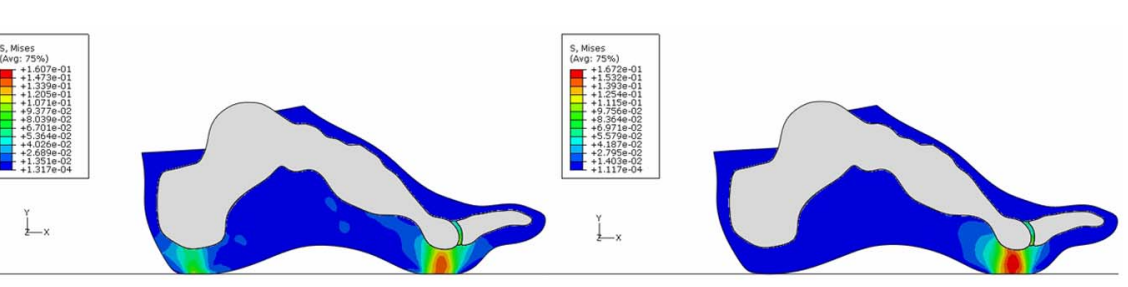

$\mathrm{t}=0.23$ (s)

$\mathrm{t}=0.27(\mathrm{~s})$

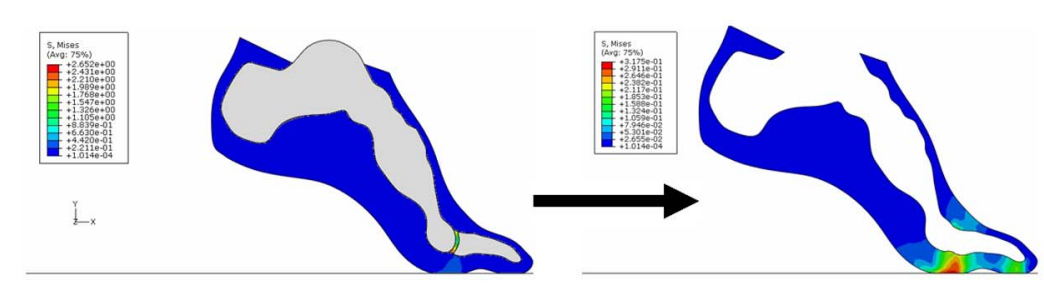

$\mathrm{t}=0.43$ (s)

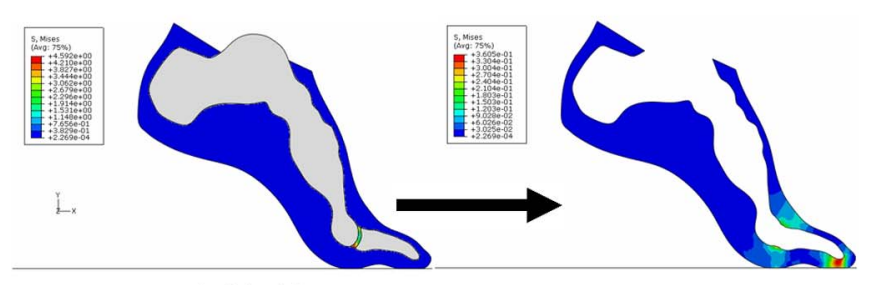

$\mathrm{t}=0.44(\mathrm{~s})$

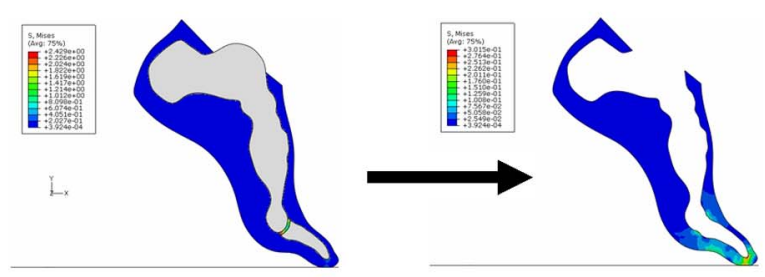

$\mathrm{t}=0.45(\mathrm{~s})$

Figure 4. Predicted von Mises stress distribution at 10 representative instants of time over whole stance phase. doi:10.1371/journal.pone.0079424.g004

In this study, the foot was allowed to move freely with respect to the ground without any predefined kinematic constraints. The foot-ground interaction was modelled as a kinematical contact with friction. This offers an alternative approach to modelling the dynamic foot-ground contact. It has the benefit that it also estimates the dynamic contact stress distribution, which cannot be obtained using the discrete spring [4] or uni-lateral rigid contact [8] methods. By incorporating these features into a forward dynamics musculoskeletal model, this foot model could also be used to conduct the simultaneous simulation of soft tissue mechanics and musculoskeletal dynamics [19,20]. This could help alleviate a major problem of many gait simulations: unrealistic foot-substrate mechanical interactions and poor matches with experimental ground reaction force data.

Direct comparison of the results of our model with previous static or quasi-static FE foot models is difficult because appreciably different loading and boundary conditions have been used in the previous studies. However, some apparent differences are found in our analysis when comparing the results of the dynamic FE simulation with the results of the quasi-static simulation based on the same foot model described in this study. The prediction accuracy of the peak plantar pressures has been improved by $10 \%$ in the heel medial region in the early stance (at $6 \%$ of the stance phase, just after heel strike) and by $33 \%$ in the $1^{\text {st }}$ metatarsal region in the middle stance (at $40 \%$ of the stance phase, shortly after metatarsal head strike) respectively, by using the dynamic FE simulation. This strongly suggests that the dynamic formulation of the FE foot model may have some advantages over the quasi-static formulation in predicting the stress and strain conditions for dynamic motions especially when ground-foot impacts are involved. The small discrepancy produced in the late stance may be explained by the excessive toe rotation generated due to the lack of the modelling of individual ankle plantarflexor muscles. 

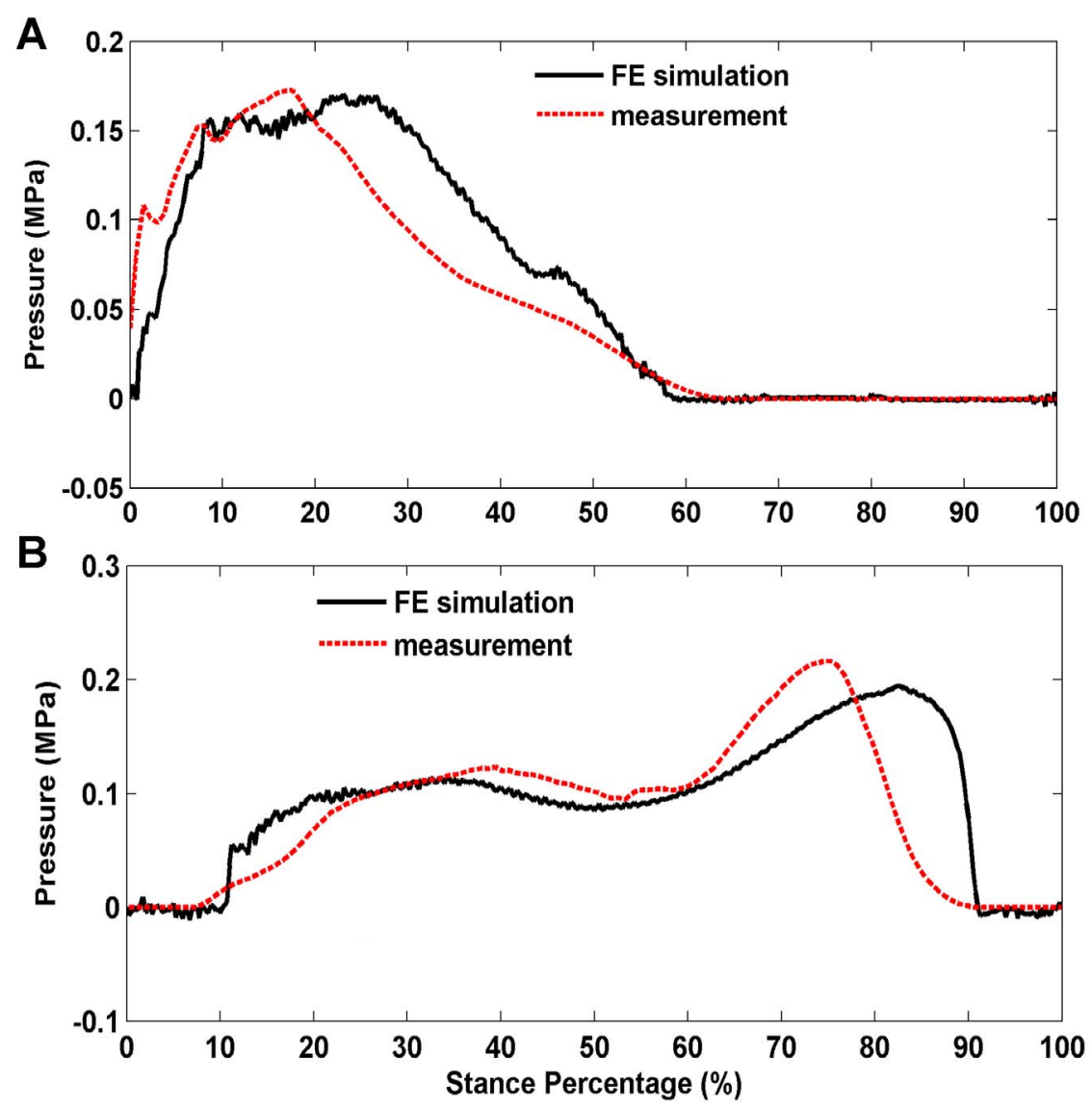

C

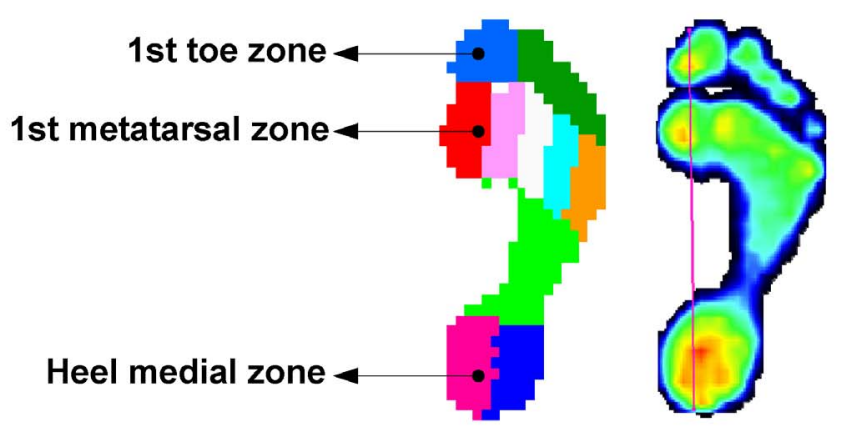

Figure 5. The calculated plantar pressure changes over the whole stance phase compared to the measurement data. (A) in heel medial region. (B) in the first metatarsal head region. The foot-ground contact area was divided into ten zones in the RSscan pressure plate system (C), so the zonal pressures could be directly calculated.

doi:10.1371/journal.pone.0079424.g005

Moreover, the close agreement of our predicted ground reaction forces, CoP displacement and foot bone motions with the individualized measurement data indicates that the dynamic FE model proposed in this study made an encouraging attempt towards a more realistic and robust approach for simulating human foot dynamics than the static or quasi-static methods. Our ongoing work involves more accurate representation of the soft tissues using non-linear isotropic viscoelastatic material, realistic anatomy of the complex 3D foot structures and modelling the contractions of individual muscle groups [40]. This would lead to a better understanding of the dynamic behaviors and the internal loading conditions of the foot musculoskeletal structures and thereby facilitate clinical diagnosis, footwear design and injury prevention.

\section{Conclusion}

A subject-specific dynamic finite element foot model was constructed and tested in this study, which demonstrated the potential to provide a more rigorous method for simulating human foot dynamics than previous static or quasi-static FE simulations, and may lead to a better understanding of the structural dynamics of the human foot musculoskeletal system during normal and even pathological functioning. Our ongoing work involves the extension of the current FE foot model to a fully muscle-driven dynamic three dimensional FE musculoskeletal model with detailed 
Table 3. The calculated peak pressure in different foot contact regions using both the dynamic FE simulation and the quasi-static FE simulation compared to the recorded pressure plate data.

\begin{tabular}{lllll}
\hline \multicolumn{1}{l}{ Time instant } & & & \\
& Contact zone & Peak pressure (MPa) & & Measurement \\
\cline { 2 - 5 } & & Dynamic simulation & Quasi-static simulation & 0.14 \\
\hline $0.03 \mathrm{~s}$ & heel medial & 0.13 & 0.12 & - \\
& $1^{\text {st }}$ metatarsal & - & - & - \\
$0.19 \mathrm{~s}$ & $1^{\text {st }}$ toe & - & - & 0.060 \\
& heel medial & 0.090 & 0.070 & 0.12 \\
$0.44 \mathrm{~s}$ & $1^{\text {st }}$ metatarsal & 0.11 & 0.070 & - \\
& $1^{\text {st }}$ toe & - & - & - \\
& heel medial & - & - & 0.00 \\
\hline & $1^{\text {st }}$ metatarsal & 0.010 & 0.010 & 0.030 \\
\hline
\end{tabular}

doi:10.1371/journal.pone.0079424.t003

representation of all major anatomical structures and also the investigation into the foot dynamics and structural responses during different motor activities.

\section{Acknowledgments}

We thank the Structure \& Motion Lab, Royal Veterinary College, for help with the gait measurements, and also the members at School of

\section{References}

1. Ker RF, Bennett MB, Bibby SR, Kester RC, Alexander RM(1987) The Spring in the Arch of the Human Foot. Nature 325: 147-149. doi:10.1038/325147a0.

2. Carrier DR, Heglund NC, Earls KD(1994) Variable Gearing During Locomotion in the Human Musculoskeletal System. Science 265: 651-653. doi:10.1126/science.8036513.

3. Ren L, Howard D, Ren LQ, Nester C, Tian LM(2008) A Phase-dependent Hypothesis for Locomotor Functions of Human Foot Complex. J Bionic Eng 5:175-180. doi:10.1016/S1672-6529(08)60022-0.

4. Scott SH, Winte DA(1993) Biomechanical Model of the Human Foot: Kinematics and Kinetics during the Stance Phase of Walking. J Biomech 26:1091-1104. doi:10.1016/S0021-9290(05)80008-9.

5. Gefen A, Megido-Ravid M, Itzchak Y, Arcan M(2000) Biomechanical Analysis of the Three-dimensional Foot Structure during Gait: A Basic Tool for Clinical Applications. J Biomech Eng 122: 630-639. doi:10.1115/1.1318904.

6. Carson MC, Harrington ME, Thompson N, O'Connor JJ, Theologis TN (2001) Kinematic Analysis of a Multi-segment Foot Model for Research and Clinical Applications: A Repeatability Analysis. J Biomech 34:1299-1307. doi:10.1016/ S0021-9290(01)00101-4.

7. Nester CJ, Liu AM, Ward E, Howard D, Cocheba J, et al.(2007) In Vitro Study of Foot Kinematics Using A Dynamic Walking Cadaver Model. J Biomech 40:1927-1937. doi:10.1016/j.jbiomech.2006.09.008.

8. Ren L, Howard D, Ren LQ Nester CJ, Tian LM (2010) A Generic Analytical Foot Rollover Model for Predicting Translational Ankle Kinematics in Gait Simulation Studies. J Biomech 43: 194-202. doi:10.1016/j.jbiomech.2009. 09.027.

9. Crompton RH, Pataky TC, Savage R, D'Août K, Bennett MR, et al. (2012) Human-like External Function of the Foot, and Fully Upright Gait, Confirmed in the 3.66 Million Year Old Laetoli Hominin Footprints by Topographic Statistics, Experimental Footprint-formation and Computer Simulation. J R Soc Interface, 9: 707-719. doi:10.1098/rsif.2011.0258.

10. Cheung JT, Nigg BM (2008) Clinical Applications of Computational Simulation of Foot and Ankle. Sport Orthop Traumatol 23:264-271. doi:10.1016/ j.orthtr.2007.11.001.

11. Lemmon D, Shiang TY, Hashmi A, Ulbrecht JS, Cavanagh PR (1997) The Effect of Insoles in Therapeutic Footwear: A Finite Element Approach. J Biomech 30: 615-620, doi:10.1016/S0021-9290(97)00006-7.

12. Patil KM, Braak LH, Huson A (1996) Analysis of Stresses in Two-dimensional Models of Normal and Neuropathic Feet. Med Biol Eng Comput 34: 280-284. doi: $10.1007 / \mathrm{BF} 02511238$.

13. Jacob S, Patil KM, Braak LH, Huson A (1996) Stresses in a 3-D Two Arch Model of A Normal Human Foot. Mech Res Commun 23:387-393. doi: 10.1016/0093-6413(96)00036-5
Mechanical, Aerospace and Givil Engineering, University of Manchester for their comments and discussions about this work.

\section{Author Contributions}

Conceived and designed the experiments: LR. Performed the experiments: ZHQ LR. Contributed reagents/materials/analysis tools: YD JRH LQR. Wrote the paper: ZHQ LR. Finite Element Modelling: ZHQ LR.

14. Jacob S, Patil KM (1999) Three-dimensional Foot Modeling and Analysis of Stresses in Normal and Early Stage Hansen's Disease with Muscle Paralysis. J Rehabil Res Dev 36: 252-263.

15. Cheung JT, Zhang M, An KN (2004) Effects of Plantar Fascia Stiffness on the Biomechanical Responses of the Ankle-foot Complex. Clin Biomech 19: 839 846. doi:10.1016/j.clinbiomech.2004.06.002.

16. Cheung JT, Zhang M, Leung AK, FanYB (2005) Three-dimensional Finite Element Analysis of the Foot during Standing-A Material Sensitivity Study. J Biomech 38: 1045-1054. doi:10.1016/j.jbiomech.2004.05.035.

17. Cheung JT, Zhang M (2008) Parametric Design of Pressure-relieving Foot Orthosis Using Statistics-based Finite Element Method. Med Eng Phys 30: 269 277. doi:10.1016/j.medengphy.2007.05.002.

18. Wu LJ (2007) Nonlinear Finite Element Analysis for Musculoskeletal Biomechanics of Medial and Lateral Plantar Longitudinal Arch of Virtual Chinese Human after Plantar Ligamentous Structure Failures. Clin Biomech 22: 221-229. doi:10.1016/j.clinbiomech.2006.09.009.

19. Halloran JP, Erdemir A, van den Bogert AJ (2009) Adaptive Surrogate Modeling for Efficient Coupling of Musculoskeletal Control and Tissue Deformation Models. J Biomech Eng 131: 011014. doi:10.1115/1.3005333.

20. Halloran JP, Ackermann M, Erdemir A, van den Bogert AJ (2010) Concurrent Musculoskeletal Dynamics and Finite Element Analysis Predicts Altered Gait Patterns to Reduce Foot Tissue Loading. J Biomech, 43: 2810-2815. doi:10.1016/j.jbiomech.2010.05.036

21. Yarnitzky G, Yizhar Z, Gefen A (2006) Real-time Subject-specific Monitoring of Internal Deformations and Stresses in the Soft Tissues of the Foot: A New Approach in Gait Analysis. J Biomech 39: 2673-2689. doi:10.1016/j.jbiomech. 2005.08.021.

22. Shin J, Yue N, Untaroiu CD (2012) A Finite Element Model of the Foot and Ankle for Automotive Impact Applications. Ann Biomed Eng 40: 2519 2531.doi: 10.1007/s10439-012-0607-3

23. Hannah I, Harland A, Price D, Lucas T (2012) Centre of Pressure Output in a Kinematically Driven Finite Element Footstrike Model. Procedia Eng 34: 278283.

24. Isvilanonda V, Dengler E, Iaquinto JM, Sangeorzan BJ, Ledoux WR (2012) Finite Element Analysis of the Foot: Model Validation and Comparison Between Two Common Treatments of the Clawed Hallux Deformity. Clin Biomech 27: 837-844. doi:10.1016/j.clinbiomech.2012.05.005

25. Dai XQ Li Y, Zhang M, Cheung JT (2006) Effect of Sock on Biomechanical Responses of Foot during Walking. Clin Biomech 21: 314-321. doi:10.1016/ j.clinbiomech.2005.10.002.

26. ABAQUS User's Manual (version 6.8), 2008, Dassault Systèmes Simula Corp, Providence, RI, USA. 
27. Chen WP, Tang FT, Ju CW (2001) Stress Distribution of the Foot during Midstance to Push-off in Barefoot Gait: A 3D Finite Element Analysis. Clin Biomech 16: 614 620. doi:10.1016/S0268-0033(01)00047-X.

28. Ren L, Jones RK, Howard D (2008) Whole Body Inverse Dynamics over A Complete Gait Cycle Based Only on Measured Kinematics. J Biomech 41 :2750-2759. doi:10.1016/j.jbiomech.2008.06.001

29. Jenkyn TR, Nicol AC (2007) A Multi-segment Kinematic Model of the Foot with A Novel Definition of Forefoot Motion for Use in Clinical Gait Analysis during Walking. J Biomech 40: 3271-3278. doi:10.1016/j.jbiomech.2007.04. 008.

30. Ren L, Jones R, Howard D (2005) Generalized Approach to Three-dimensional Marker-based Motion Analysis of Biomechanical Multi-segment Systems. Proc International Society of Biomechanics XXth Congress, Cleveland, Ohio, USA.

31. Cappozzo A, Catani F, Croce UD, Leardini A (1995) Position and Orientation in Space of Bones during Movement: Anatomical Frame Definition and Determination. Clin Biomech 10: 171-178. doi:10.1016/0268-0033(95)91394T.

32. Cahouët V, Luc M, David A (2002) Static Optimal Estimation of Joint Acceleration for Inverse Dynamics Problem Solution. J Biomech 35: 1507-1513. doi:10.1016/S0021-9290(02)00176-8.

33. Kuo AD (1998) A Least Squares Estimation Approach to Improving the Precision of Inverse Dynamics Computations. J Biomech Eng 120:148-159. doi:10.1115/1.2834295.
34. Erdemir A, Viveiros ML, Ulbrecht JS, Cavanagh PR (2006) An Inverse Finiteelement Model of Heel-pad Indentation. J Biomech 39:1279-1286.doi: 10.1016/j.jbiomech.2005.03.007.

35. Ker RF (1996) The Time-dependent Mechanical Properties of the Human Heel pad in the Context of Locomotion. J Exp Biol 199:1501-1508.

36. Hicks JH (1954) The Mechanics of the Foot II. The Plantar Aponeurosis and the Arch. J Anat 88: 25-30.

37. Chu TM, Reddy NP, Padovan J (1995) Three-dimensional Finite Element Stress Analysis of the Polypropylene, Ankle-foot Orthosis: Static Analysis. Med Eng Phys 17: 372-379. doi:10.1016/1350-4533(95)97317-I.

38. Hsu YC, Gung YW, Shih SL, Feng CK, Wei SH, et al. (2008) Using An Optimization Approach to Design An Insole for Lowering Plantar Fascia StressA Finite Element Study. Ann Biomed Eng 36: 1345-1352. doi: 10.1007/s10439008-9516-x.

39. Miller-Young JE, Duncan NA, Baroud G (2002) Material Properties of the Human Calcaneal Fat Pad in Compression: Experiment and Theory. J Biomech 35: 1523-1531. doi:10.1016/S0021-9290(02)00090-8.

40. Qian ZH, Ren L, Ren LO Boonpratatong A (2010) A Three-Dimensional Finite Element Musculoskeletal Model of the Human Foot Complex. Proc. of the $6^{\text {th }}$ World Congress of Biomechanics, Singapore, 297-300. 